\title{
Baylor news
}

U.S. News \& World Report ranks two Baylor Scott \& White Health hospitals in Texas' top 10

Seven Baylor Scott \& White Health (BSWH) hospitals have been ranked some of the best in Texas for 2014-2015 by U.S. News \& World Report in its annual Best Hospitals rankings. Two BSWH hospitals have ranked in the top 10 in the state of Texas. Baylor University Medical Center at Dallas is ranked No. 1 in the Dallas metro area and No. 2 in Texas. Scott \& White Memorial Hospital is ranked №. 10 in Texas, moving up eight spots from the previous year.

"We are so proud to see the two flagship hospitals of the new Baylor Scott \& White Health both rank in the top 10 in the state," said Joel Allison, CEO, BSWH. "When we merged Baylor Health Care System and Scott \& White Healthcare in 2013, we knew we were combining two exemplary systems known for high-quality care. These 2014-2015 U.S. News rankings are proof, as our new system now includes a total seven ranked hospitals throughout North and Central Texas."

Baylor University Medical Center at Dallas, which has been included in the U.S. News \& World Report rankings for more than 20 years, was ranked nationally in 2014 for six medical specialties: gastroenterology and Gl surgery (no. 15 in the nation), diabetes and endocrinology (no. 30 in the nation), pulmonology (no. 35 in the nation), nephrology (no. 38 in the nation), neurology and neurosurgery (no. 40 in the nation), and orthopedics (no. 41 in the nation). The hospital is also high performing in cancer, cardiology and heart surgery, geriatrics, gynecology, urology, and ear, nose, and throat.

For 2014-2015, U.S. News evaluated hospitals in 16 adult specialties and ranked the top 50 in most of the specialties. Just 3\% of the nearly 5000 hospitals that were analyzed for Best Hospitals 2014-2015 earned national ranking in even one specialty.

In its metro-area rankings, U.S. News and World Report gives consumers information about high-performing hospitals in their backyard. This year, U.S. News and World Report ranked six BSWH hospitals in the Dallas metro area:

- Baylor University Medical Center at Dallas: no. 1

- Baylor Regional Medical Center at Plano: no. 6

- Baylor Medical Center at Irving: no. 7
- Baylor All Saints Medical Center at Fort Worth: no. 8

- Baylor Regional Medical Center at Grapevine: no. 8

- Baylor Institute for Rehabilitation: no. 12

\section{Baylor Scott and White Health, eight other systems form Texas Care Alliance}

BSWH, the largest nonprofit health system in Texas, is one of nine Texas health systems joining forces to share clinical and administrative data to improve quality and efficiency, lower health care costs, and accelerate medical innovation in patient care. The Texas Care Alliance (TCA) was founded by BSWH and Trinity Mother Frances Hospitals and Clinics of Tyler in March 2013 to facilitate the coordination and delivery of health care services that are people centered and physician driven to improve the overall health of a defined population. TCA also includes Community Hospital Corporation, Plano; Good Shepherd Health System, Longview; Hendrick Health System, Abilene; Medical Center Health System, Odessa; Midland Memorial Hospital, Midland; Shannon Health System, San Angelo; and United Regional Health Care System, Wichita Falls. The systems' service areas cover 193 Texas counties. TCA members have $20 \%$ of the state's hospital beds with more than 6800 employed or affiliated physicians.

"Now more than ever, it is critical for community-based health care providers to collaborate and accelerate change," said Joel Allison, BSWH CEO and TCA chairman of the board. "The TCA exists to support providers who are stepping up to the challenges of health care now and in the future. We are going to demonstrate innovative, value-based methods for reducing costs and improving quality in a post-health care reform world."

To qualify for payment based on quality of care-which will account for two-thirds of health care revenue in 5 years-providers are employing powerful analytical tools for population health and risk management. TCA will be using Explorys, a data platform that is a spinoff of the Cleveland Clinic. The Explorys network includes more than 310 hospitals and 220,000 practitioners. Explorys will facilitate data sharing between practitioners, payers, and health systems. The data will allow health care providers to build a composite view of a population's health to meet care needs, improve quality, and assess risk of disease. TCA will be able to harness the data from tens of millions of patient encounters from these health systems and compare them to the TCA experience as a whole as well as at the individual hospital and practitioner level to minimize uncertainty and potential study bias as better care and improved health at a lower cost is achieved.

\section{Baylor Carrollton receives game- changing imaging technology}

Installation of the AiroMobile Intraoperative computed tomography (CT) system-only the second in the world-delivers gamechanging technology to Baylor Medical Center at Carrollton patients. Baylor Carrollton's new imaging technology introduces mobile CT capabilities during surgery with real-time, highquality patient images to aid operating room (OR) decision-making.

This imaging system offers a combination of mobility and speed previously unachievable in the $\mathrm{OR}$ setting. The CT scanner isn't limited to one $\mathrm{OR}$ like many systems currently employed in the industry. It can be easily transported to any of the nine ORs and other patient care locations in the hospital. The equipment is extremely versatile-adapting to a variety of surgical setups and eliminating the need to move the patient in order to get images. CT images can be taken and developed in less than a minute or two in most cases. Alternative options use either twodimensional $\mathrm{x}$-rays or magnetic resonance scans, which can sometimes take 30 to 40 minutes to develop.

"This combination is a game changer. In the past, only one or maybe two of these capabilities were available to use along with image-guided surgery systems in an $\mathrm{OR}$. The advantages of combining all of these in one allows the surgical team to be extremely precise, which is critically important when you are talking about complicated spine and brain surgeries," said Michael Turner, MD, medical director of neurosurgery at Baylor Carrollton.

"We are excited to be one of the first hospitals not only in the country, but in the world, to use this new technology in our operating rooms at Baylor Carrollton. We hope this innovative imaging system will further enhance the quality care we are able to offer our patients," said Michael Sanborn, hospital president. 


\section{ACCOLADES}

The nation's oldest and most prestigious surgical organization, the American Surgical Association, has voted to approve Marlon Levy, MD, FACS, for membership. This is an esteemed honor, not only because of the ASA's premier reputation, but also because the ASA caps membership at 400.

David J. Ballard, MD, PhD, chief quality officer for BSWH, has been appointed to the National Advisory Council for Healthcare Research and Quality. The 21-member panel provides advice and recommendations to the secretary of the US Department of Health and Human Services and the director of the Agency for Healthcare Research and Quality on priorities for national health services research.

The cancer programs at Baylor Regional Medical Center at Plano and the Cancer Institute at Scott and White Memorial Hospital Temple-Central Texas were one of the top 74 programs in the US, according to the American College of Surgeons Commission on Cancer. These programs received a 2013 Outstanding Achievement award from the cancer commission, signifying that they are among "the best of the best," according to Daniel P. McKellar, MD, FACS, chair of the commission.

In an effort to help patients make informed choices, Consumer Reports has begun rating hospitals based on outcomes of heart surgery. The Heart Hospital Baylor Plano scored in the top 15. Based on data from the Society of Thoracic Surgeons, Consumer Reports rates more than 400 hospitals in 45 states, plus Washington, DC, and Puerto Rico. Although more than 1000 hospitals report data to the Society of Thoracic Surgeons, only about 400 elected to share their information with Consumer Reports.

The Joint Commission, in conjunction with The American Heart Association/American Stroke Association, recently recognized Baylor All Saints Medical Center at Fort
Worth with Advanced Certification for Primary Stroke Centers. Achievement of Primary Stroke Center Certification signifies an organization's dedication to fostering better outcomes for patients.

Baylor Health Care System's Supportive and Palliative Care Program was one of three in the United States to win the American Hospital Association's 2014 Circle of Life Award: Celebrating Innovation in Palliative and End-of-Life Care. Baylor was chosen by a selection committee made up of leaders from medicine, nursing, social work, ethics, and health administration. The judges cited the success of the Baylor's Supportive and Palliative Care Program in promoting the culture of palliative care for patients and families facing the most serious illness throughout the system. Robert L. Fine, $M D$, and Martha Philastre, MBA, direct the programs at Baylor Health Care System, now part of BSWH.

\section{UPCOMING CME PROGRAMS}

The A. Webb Roberts Center for Continuing Education of Baylor Health Care System is offering the following programs:

North Texas Regional Cardiovascular Conference, November 15, 2014, at the Gaylord Texan Resort and Convention Center, Grapevine, Texas

41st Annual Williamsburg Conference on Heart Disease, December 7-9, 2014, in Williamsburg, Virginia

18th Annual Tyler Breast Cancer Conference, March 27-28, 2015, at the Harvey Convention Center, Tyler, Texas

6th Annual Latest Advances in Ischemic and Hemorrhagic Stroke Therapy, May 16, 2015, at the Dallas Marriott City Center, Dallas, Texas

For more information, call 214.820.2317 or visit www.cmebaylor.org.

- Hospital equipment finds second life in Liberia, thanks to Faith in Action Initiatives

Phebe Hospital in Liberia, Africa, has been struggling to find the resources to care for the 350,000 people who rely on the 500 -bed hospital for their medical care. Now, thanks to a delivery of hospital beds, equipment, and other supplies from Faith in Action Initiatives (FIAI), the hospital is making a dent in the need.
FIAl, a BSWH-based program, relies on connections and collaboration to accomplish its mission of strengthening the spiritual lives of staff and empowering medical mission work. As an offshoot of its primary mission, FIAl also provides "second-life resources" — retired medical equipment and supplies that are recycled and put to use in hospitals, clinics, and other nonprofit agencies. By using the synergy of volunteers, churches, and other faith-based agencies, FIAI has a global reach far beyond its size.

\section{RECENT GRANTS}

- Mechanisms of $B$ cell responses in autoimmune disease: M12-ALE05-BRI Principal investigator: Virginia Pascual, MD Sponsor: Duke University/National Institutes of Health

Funding: $\$ 334,737$ Award period: 5/1/2014-4/30/2015

- JC virus and human colorectal neoplasia Principal investigator: C. Richard Boland, MD Sponsor: National Institutes of Health Funding: $\$ 259,352$

Award period: 2/1/2014-1/30/2015

- Methylation biomarker development for noninvasive detection of colorectal cancer

Principal investigator: Ajay Goel, PhD Sponsor: National Institutes of Health Funding: $\$ 327,289$

Award period: 5/21/2014-4/30/2015

- Pain management after thoracostomy: is EXPAREL better than lidocaine Principal investigator: Laura Petrey, MD Sponsor: Pacira Pharmaceutical Inc. Funding: $\$ 23,605$

Award period: 2/27/2014-2/26/2015 


\section{PHILANTHROPY NOTES}

- $\$ 1$ million gift from Hal and Diane Brierley is far-reaching

Long-time supporters Diane and Hal Brierley have made a generous $\$ 1$ million gift through the Hal and Diane Brierley Foundation. The gift will benefit diverse areas of need across Baylor Health Care System. This latest gift will support the Memory Center at Baylor University Medical Center at Dallas, Baylor's service animal training program, and multiple myeloma research, for which the Brierleys have a personal connection.

Ryan Anthony, a world-renowned trumpet musician, has held the Principal/Diane and Hal Brierley Chair at the Dallas Symphony Orchestra since 2008. In 2013, Ryan was diagnosed with multiple myeloma. After researching treatment centers across the country, Ryan elected to have his bone marrow transplant and treatment at Baylor Health Care System, where he felt he would receive the best care and outcome. Ryan is currently in remission but remains passionate about the need to find a cure. He is helping to organize a fundraiser to support multiple myeloma research. The event will include performances by world-class trumpet players and be held at the Meyerson Symphony Center in March 2015.

\section{- Good Morning America co-host and breast cancer survivor, Amy Robach, to speak at 15th annual Celebrating Women luncheon}

Breast cancer survivor, ABC News correspondent, and "Good Morning America" cohost Amy Robach will be the featured speaker at the 15th annual Baylor Health Care System Foundation Celebrating Women luncheon on Friday, October 24, at the Hilton Anatole Hotel in Dallas. As part of "Good Morning America Goes Pink Day" in honor of Breast Cancer Awareness Month last October, Amy had her first mammogram at age 40 and agreed to participate in the first-ever on-air mammogram. Following the mammogram, Amy received lifealtering news that she had been diagnosed with breast cancer. In the weeks that followed, she underwent a double mastectomy and recently completed treatment for the disease.

Since the first Celebrating Women luncheon in 2000, more than $\$ 21$ million has been raised to support Baylor Health Care System's 50+year fight against breast cancer. Donations to Celebrating Women have supported advanced diagnostic equipment, innovative clinical research, and safe, quality, compassionate care for Baylor Health Care System patients and families. Approximately 1200 passionate men and women attend the Celebrating Women luncheon each year in a show of support for Baylor Health Care System efforts to fight the disease.

For information about underwriting opportunities and tickets to Celebrating Women, call 214.820.4500 or e-mail CelebratingWomen@ BaylorHealth.edu. Sponsorship and underwriting opportunities are still available; individual tickets start at $\$ 250$ and table prices start at $\$ 2500$.

\section{Gift from Alon USA bears fruit}

Thanks to a generous $\$ 100,000$ gift from Alon USA in support of the Diabetes Health and Wellness Institute (DHWI), residents in the Frazier community in South Dallas will continue to enjoy access to fresh fruits and vegetables at the Friday Farm Stand. The Farm Stand, located inside DHWI's facility, offers healthy produce options at a discounted price.

The USDA considers the Frazier community a "food desert" due to limited access to commercial grocery stores in the area. Frazier is one of the city's oldest historic neighborhoods; it also has the highest rate of diabetes and diabetes-related complications and hospitalizations of any neighborhood in Dallas. DHWI relies heavily on philanthropic support to provide services to this underserved community. The fifth annual DHWI Healthy Harvest Fun Walk/5K Run and Diabetes Expo, an awareness and fundraising initiative benefiting the Institute, is scheduled for Saturday, October 25.

For more information about DHWI, please contact Amy Monday at 214.820.4771 or Amy. Monday@BaylorHealth.edu. For more information about sponsorship opportunities for the DHWI Healthy Harvest event, please contact Courtney Brown at 214.820.7410 or Courtney. Brown@BaylorHealth.edu.

$\$ 500,000$ Fetal Care Challenge grant will help Baylor's tiniest patients

Thanks to a $\$ 500,000$ gift from an anonymous donor, Baylor Health Care System Foundation has been challenged to raise an additional $\$ 500,000$ to support the work of the Fetal Care Center at Baylor University Medical Center at Dallas, under the medical direction of Kevin Magee, MD.

Known as the Fetal Care Challenge, the Foundation has a goal of raising an additional $\$ 500,000$ to make the Fetal Care Center at Baylor Dallas the first center in North Texas to provide correction of spina bifida while the baby is still in the mother's womb. In addition, gifts to the Fetal Care Challenge will support research to develop procedures to correct other significant medical issues faced by the unborn. This Fetal Care Challenge will also provide technologically advanced imaging and monitoring capability for the 30-bed maternal fetal care unit.

For information on how you can support these or other initiatives at Baylor, please contact the Foundation at 214.820.3136. 